\title{
REVIEW
}

\section{MicroRNAs and respiratory diseases}

\author{
Hitasha Rupani, Tilman Sanchez-Elsner and Peter Howarth
}

\begin{abstract}
MicroRNAs (miRNAs) are a family of endogenous, small, noncoding RNA molecules that modulate physiological and pathological processes by post-transcriptional inhibition of gene expression. They were first recognised as regulators of development in worms and fruitflies. In recent years extensive research has explored their pivotal role in the pathogenesis of human diseases. Over 1,000 human miRNAs have been discovered to date; however, the biological function and protein targets for the majority remain to be uncovered. Within the respiratory system, miRNAs are important in normal pulmonary development and maintaining lung homeostasis. Recent studies have also begun to reveal that altered miRNA expression profiles may be associated with pathological processes within the lung and lead to the development of various pulmonary diseases, ranging from inflammatory diseases to lung cancers. Advancing our understanding of the role of miRNAs in the respiratory system will help provide new perspectives on disease mechanisms and reveal intriguing therapeutic targets and diagnostic markers for respiratory disorders.
\end{abstract}

KEYWORDS: Adaptive immune response, asthma, cigarette smoke, innate immune response, lung cancer, microRNA regulation

$\mathbf{T}$ he human genome was once predicted to contain $>100,000$ protein-coding genes, based on the assumption that as complex organisms we would have a large number of genes. We now know there are $<30,000$ proteincoding genes in the human genome - only slightly more than the unsophisticated nematode Caenorhabditis elegans [1]. However, over the last decade we have also learned that protein-coding genes (which are transcribed to mRNA) comprise only $2 \%$ of the human genome, and the vast majority is transcribed as non-protein-coding RNA (ncRNA) [2]. These ncRNAs are involved in many vital roles within our bodies, including protein synthesis (transfer RNAs and ribosomal RNAs), splicing of mRNA (spliceosomal RNAs) and regulation of gene expression (microRNAs).

MicroRNAs (miRNAs) are small ncRNA molecules ( $\sim 22$ nucleotides (nt) in length) that constitute a broad layer of gene regulation. They usually act as endogenous repressors of gene activity by translational repression and mRNA degradation. They have been found to be crucial to several biological processes, including cell proliferation, apoptosis and differentiation. Indeed, computational analyses suggest that miRNAs may regulate $>60 \%$ of the human genome [3,4]. Although they were first discovered in 1993 in the nematode C. elegans [5], the term "microRNA" was not introduced until
2001 [6]. Over 1,000 human miRNA sequences are now recognised, many of which are highly conserved in other organisms, suggesting that they represent a relatively old and important regulatory pathway [7-9]. miRNAs are distinct from the better-known small interfering RNAs (siRNAs) that knock down transcription of single genes, as a single miRNA may target several hundred different mRNAs. Table 1 summarises some of the differences between miRNAs and siRNAs.

Several human diseases have been associated with deregulated miRNA expression, ranging from metabolic and inflammatory diseases to malignancy [11-15]. miRNAs are also pivotal to both adaptive and innate immunity, with regulatory effects on cell differentiation and immunological function [16]. However, despite our increasing awareness about their role in human diseases, much is yet to be learnt about their functional targets, biological relevance and multifactorial role in development and disease. This review focuses on the emerging role of miRNAs in respiratory disease.

\section{MIRNA BIOGENESIS}

miRNA biogenesis involves a series of complex steps (fig. 1) which begin in the nucleus and are completed in the cytoplasm. miRNA genes are localised in the non-coding regions or introns of

\section{AFFILIATIONS}

Faculty of Medicine, Clinical and Experimental Sciences, University of Southampton, Southampton, UK.

CORRESPONDENCE

H. Rupani

MP813 Level E South Academic Block, Faculty of Medicine Southampton General Hospital Tremona Road Southampton S016 6YD UK E-mail: hitasha@doctors.org.uk

Received:

Dec 022011

Accepted after revision: June 212012

First published online:

July 212012 
TABLE 1 Differences between microRNA (miRNA) and small interfering RNA (siRNA)

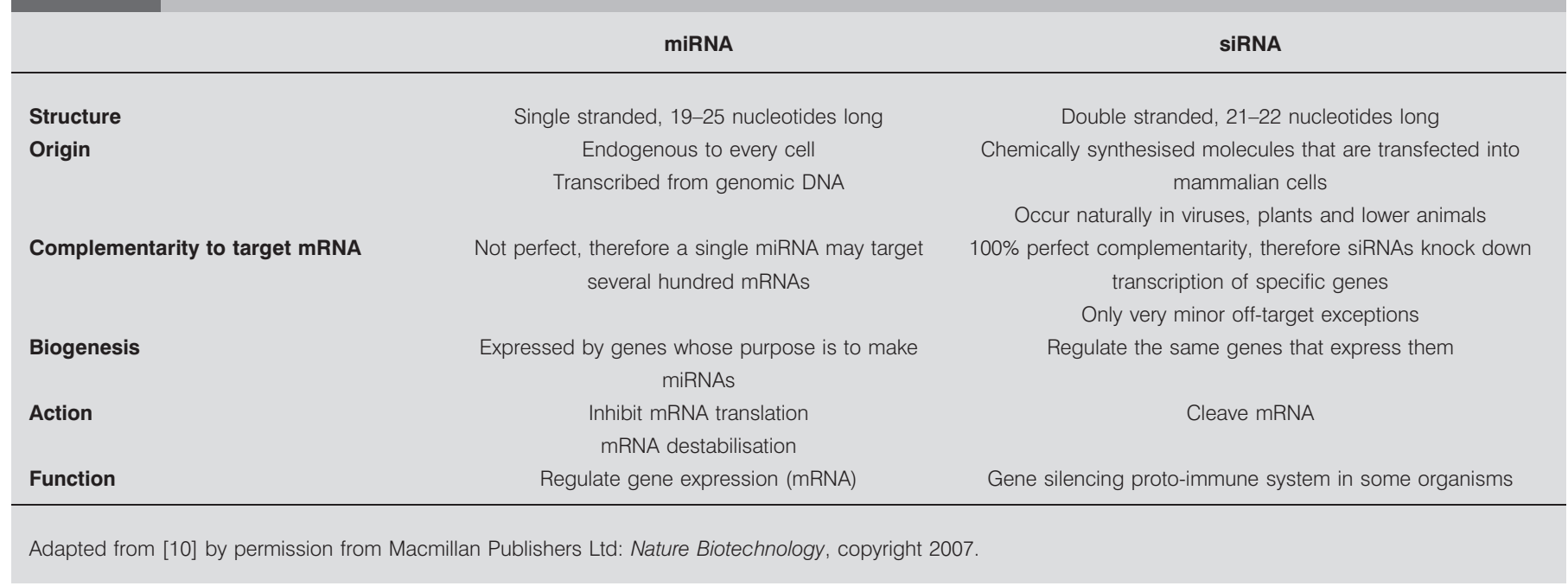

protein-coding genes in genomic DNA. Within the nucleus, primary miRNAs (pri-miRNAs; generally $>1 \mathrm{~kb}$ long) are transcribed mainly by RNA polymerase II [9]. The pri-miRNA undergoes a series of endonucleolytic steps including cleavage by a nuclear RNase III enzyme termed “Drosha" [17-19]. The

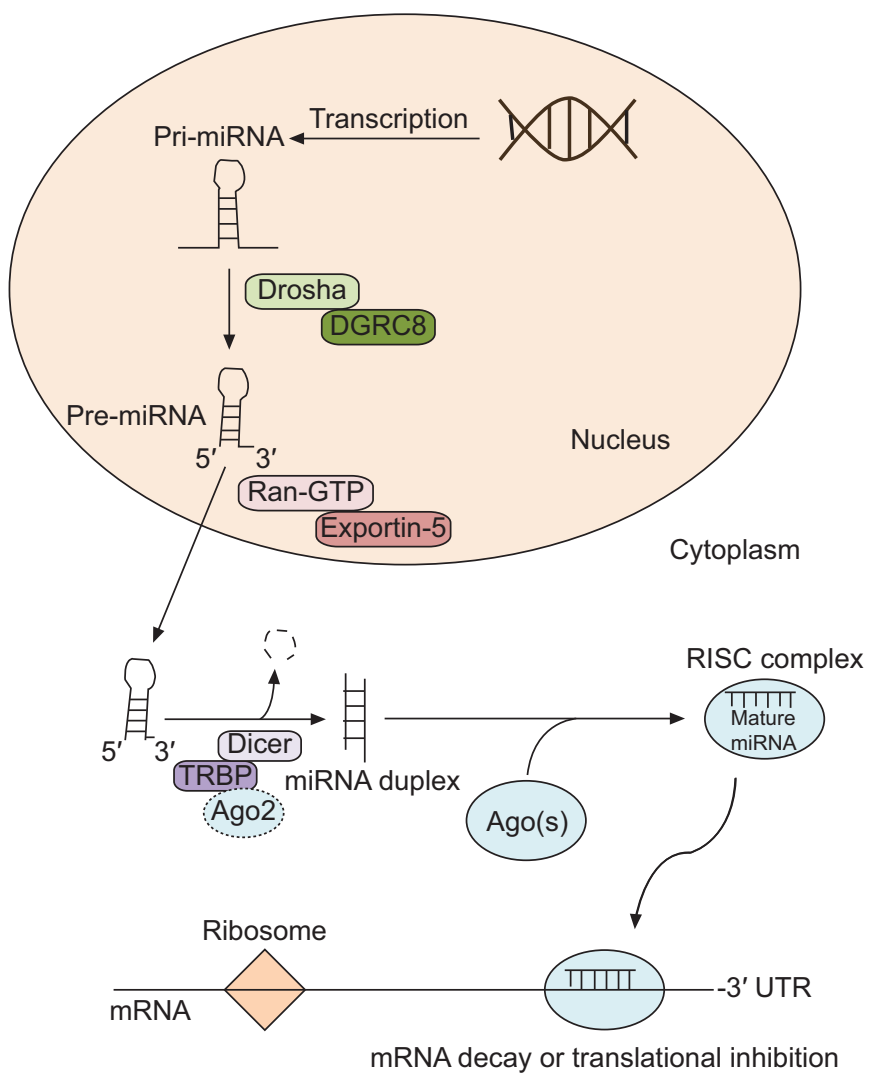

FIGURE 1. MicroRNA (miRNA) biogenesis. Pri-miRNA: primary miRNA; DGRC8: DiGeorge syndrome critical region gene 8; Ran-GTP: Ras-related nuclear protein-guanosine-5'-triphosphate; TRBP: trans-activation response RNA-binding protein; Ago: Argonaute protein; RISC: RNA-induced silencing complex; UTR: untranslated region. resulting 70-nt pre-miRNA is actively transported into the cytoplasm and processed into a 22-nt double-stranded miRNA by a cytoplasmic RNase III enzyme termed "Dicer". One strand of this duplex, representing the mature miRNA, is incorporated into the RNA-induced silencing complex (RISC), while the other strand is degraded. Assembled into the RISC, the miRNA regulates gene expression by either translational repression or mRNA degradation, which is dependent on sequence complementarity between the miRNA 5' region (the seed sequence) and mRNA 3' untranslated region (3'UTR) $[9,20,21]$. It has recently been shown that target mRNA degradation or destabilisation is the predominant mechanism for reduced protein output: about $84 \%$ of target repression mediated by miRNAs is due to decreased mRNA levels, which exceeds previous estimates [22].

\section{STRATEGIES FOR PROFILING MIRNA EXPRESSION AND TARGET GENE IDENTIFICATION}

miRNAs are structurally different from traditional RNAs (e.g. mRNA), and methods used to profile and quantify traditional RNAs need to be modified and adapted to study miRNAs. Early on, Northern blotting and cDNA arrays were used to study miRNAs. More recently, a range of techniques have emerged, which are described in table 2.

Quantitative PCR, first developed in 2004 [23], is commonly used to identify differences between two groups of samples, often a "normal" control and a "disease" sample. The accuracy of this assessment is crucially dependent on proper normalisation of the data in order to remove as much variation as possible between these groups except for variability that is a consequence of the disease itself [24]. Ideally, a normaliser is a single nucleic acid that is consistently expressed across all samples and demonstrates equivalent storage stability, extraction and quantification efficacy as the target miRNA. Occasionally, more than one miRNA normaliser/endogenous control is used in order to improve accuracy and robustness. For microarrays, other data-driven normalisation methods are frequently used and include mean expression normalisation and quantile normalisation [25]. Finally, when comparing sample groups, in addition to relative expression it is also 
TABLE 2 Techniques used to profile microRNA (miRNA) expression

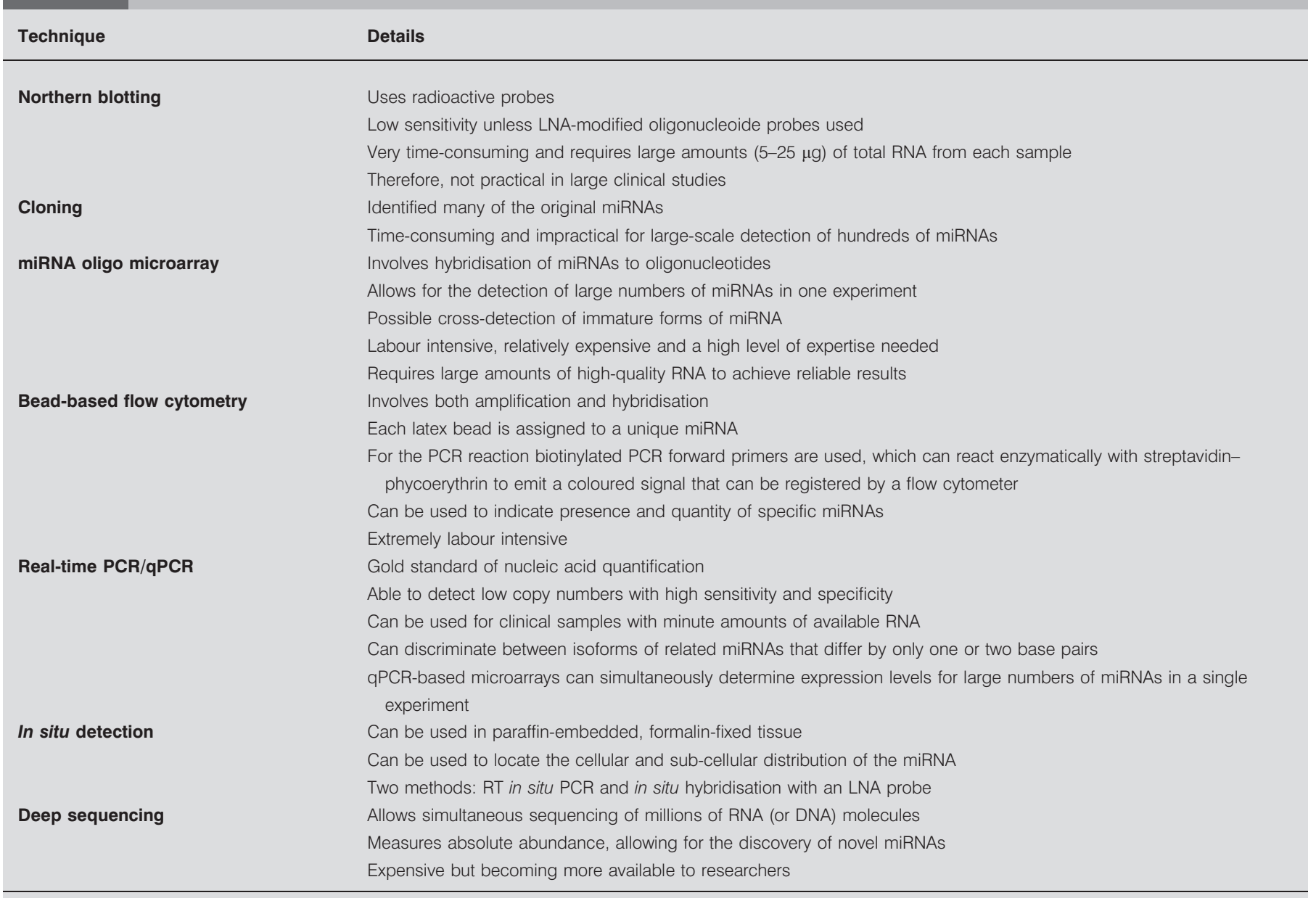

qPCR: quantitative PCR; LNA: locked nucleic acid; RT: reverse transcriptase.

useful to consider absolute miRNA levels: a change from 1,000 to 2,000 units could be argued as being more biologically relevant than a change from five to 10 units [26].

Each miRNA has hundreds of evolutionarily conserved targets and several times that number of non-conserved targets [27]. It is also recognised that multiple miRNAs can target the same gene, suggesting that it is the combination of all these activities that determines the expression of the gene and the biological significance of the interactions [28]. Computational algorithms have been the major driving force in predicting miRNA targets and most researchers use target gene-prediction databases such as PicTar, TargetScan, miRana, miRBase and miRGen [29]. Different algorithms may yield different miRNA-mRNA predictions and it is imperative that the researcher is aware that there may be false positives (pairs that are statistically significant but cannot be verified) and false negatives (true pairs that are missing from the results). It is common practice to focus on targets that are predicted by multiple algorithms. One commonly used technique for validating miRNA target genes is to either overexpress or inhibit the miRNA in the selected cell, assess whether this corresponds to a predictable change in the amount of protein encoded by the target mRNA and demonstrate that this equates to changes in biological function. To complement this and confirm a direct molecular link between the miRNA and mRNA, the 3'UTR of the target mRNA can be cloned into a luciferase reporter. The recombinant plasmid, along with the miRNA of interest, is then transfected into a cell and luciferase activity or light emission measured [30, 31] (fig. 2).

\section{REGULATION OF MIRNAS}

Most miRNAs are predicted to target many hundreds of mRNAs, simultaneously targeting multiple gene pathways to fine-tune gene expression. Therefore, multiple mechanisms have evolved to stringently regulate miRNA function at multiple levels, for example, during transcription, miRNA processing and target interaction [32]. At the transcription level, a number of regulatory factors have been identified that bind directly to the miRNA promoter elements and control their expression. For instance, the tumour suppressor gene p53 has been shown to bind to the promoter regions of the miR-34 family and activate their expression. The overexpression of miR-34 in vitro leads to cell cycle arrest and apoptosis, effects similar to those of enforced p53 expression alone [33-35]. miRNA transcription also appears to be under epigenetic control and dependent on methylation status. This is supported by the 
a)

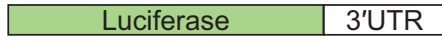

b)
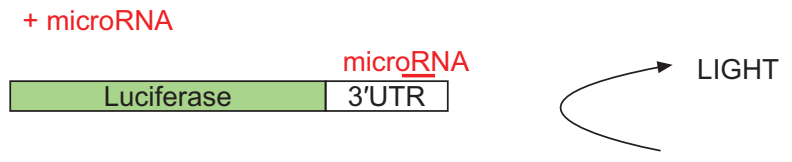

c)

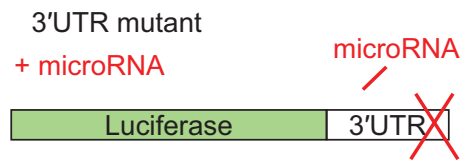

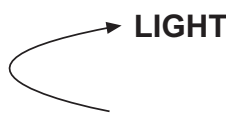

moke, pollutants and diet $[32,47,48]$. Fascinatingly, this could have implications for subsequent generations as it has been demonstrated in a murine model that a maternal high-fat diet leads to an altered expression of key miRNAs in offspring [49].

\section{INVOLVEMENT IN RESPIRATORY DISEASE}

miRNAs have been shown to be expressed in a tissue-specific and developmental stage-specific manner [50-52]. The lung has been shown to have a very specific miRNA expression profile and these miRNAs are important for lung development and in maintaining lung homeostasis [53, 54]. A global reduction in miRNAs through lung-specific deletion of Dicer leads to abnormal apoptosis and airway branching during lung development [55]. The miR-17-92 cluster (which contains miR-17, -18, -19, -20 and -92) is believed to regulate lung development because its expression is high in embryonic lung and steadily declines through development into adulthood [56]. Mice deficient in the miR-17-92 cluster develop hypoplastic lungs, while overexpression of the miR-17-92 cluster leads to the absence of terminal bronchioles, which are replaced by proliferative, undifferentiated epithelium [56, 57] In addition to their involvement in health and development, it is also apparent that deregulation of miRNAs is likely to underlie a range of pulmonary disorders.

\section{LUNG CANCER}

Lung cancer is the leading cause of cancer-related death worldwide. Despite advances in medical care and technology, 5-yr survival rates have failed to show significant improvements since the 1970s, underscoring the need for novel modalities for early detection, prognostication and targeted therapies. miRNA mis-expression has been documented in several solid and haematological malignancies. Their importance in cancer is highlighted by the observation that $\sim 50 \%$ of miRNA genes are located in cancer-associated genomic regions or fragile sites, which are frequently amplified or deleted in tumorigenesis [58]. As our understanding of miRNAs is increasing it is becoming clear that miRNAs are not only involved in lung cancer development and progression, but may also serve as biomarkers for diagnosis, prognosis and response to treatment. Two patterns of miRNA deregulation in tumours are commonly observed. The first occurs in solid tumours in which a group of miRNAs are deregulated, suggesting that these miRNAs may be involved in crucial cellular pathways such as cell-cell adhesion and signalling, cell-cycle regulation and apoptosis. Secondly, certain miRNAs are deregulated only in specific tumours, suggesting some tumour-specific micro-environment may be in place to favour particular miRNA profiles, allowing their use as biomarkers.

\section{Tumour suppressor miRNAs and oncogenic miRNAs}

The role of oncogenes and tumour suppressor genes in the pathogenesis of cancer is well known and miRNAs not only control the expression of known protein-coding oncogenes and tumour suppressors, but also act as oncogenes and tumour suppressors directly [59].

Several miRNAs have been shown to be expressed at lower levels in lung cancer when compared to normal lung. Among these, the most extensively studied for its tumour suppressor activity is the let-7 family. This was the first group of miRNAs found to be downregulated in lung cancer. Let-7 has been 
shown to negatively regulate the expression of the RAS oncogene [60] and, when administered intranasally, to reduce tumour formation in a Ras mouse model of lung cancer [61]. Overexpression of let-7 in lung cancer cell lines in vitro leads to repression of cancer cell growth [62], while reduced let-7 expression in lung cancer resection cases has been shown to be correlated with poor post-operative survival, independent of other prognostic factors, including disease stage [63]. Furthermore let-7 over-expression in lung cancer cells in vitro has been shown to suppress radio-resistance, suggesting it could also be used as a tool to augment current cancer therapy [64].

The miR-17-92 cluster has been shown to be markedly overexpressed in lung cancers, especially small cell lung cancers [65]. When over-expressed it has the ability to target multiple cellular pathways to favour tumorigenesis by enhancing cell proliferation, inhibiting apoptosis and increasing tumour vasculature [66]. These vascular effects have been related to the repression of the anti-angiogenic proteins thrombospondin- 1 and connective tissue growth factor by this miRNA cluster [67]. Conversely, inhibition of certain members of this cluster has been shown to induce apoptosis and reduce cell growth in lung cancer cell lines [68].

\section{Lung cancer biomarkers, diagnosis and prognosis}

miRNAs are particularly attractive as biomarkers because they are maintained in a stable form in accessible body fluids and their expression patterns are easily detectable within blood, saliva and urine $[69,70]$. For example, differences in the expression pattern of miRNAs in serum have been reported to correctly discriminate between healthy subjects and patients with lung cancer and colorectal cancer [71]. Within body fluids, miRNAs are thought to be free-circulating and concentrated in exosomes (small microvesicles secreted by cells including epithelial cells, lymphocytes, dendritic cells and neurons) $[72,73]$.

Recent advances in biologically directed therapies for nonsmall cell lung cancer (NSCLC) require more accurate sub-classification of NSCLC due to the serious and sometimes lifethreatening complications associated with some of the these treatments. Using formalin-fixed tissue, a miRNA-based diagnostic assay has been developed that can sub-classify NSCLC with high sensitivity (96\%), specificity $(90 \%)$ and reproducibility [74]. The sensitivity and specificity of the miRNA assay compared favourably with histological diagnosis, making it a promising tool for the future. Additionally, miRNAs can also be used to distinguish between primary lung tumours and lung metastases originating from other primary tumours [59].

Finally, miRNAs can be used to help predict prognosis and cancer survival. It has been shown in 112 patients with NSCLC that a five-miRNA signature (let-7a, miR-221, miR-372, miR137 and miR-182*) correlates with survival and cancer relapse [75]. Patients with a high risk score for this five-miRNA signature in their tumour specimens had increased cancer relapse and shortened survival, even after stratifying patients by stage or histology subgroups. Additionally, it has been demonstrated that the expression of eight miRNAs is related to the survival of patients with adenocarcinoma [76], while more recently, mature miR-21 and a single-nucleotide polymorphism in miR-196a-2 have been found to be associated with survival in NSCLC patients [77, 78].

\section{Other biological functions of relevance}

The p53 protein is a well-known major tumour suppressor and its encoding gene is mutated in about half of human cancers, while in most of the remainder the p53 pathway is inactivated. Evidence is starting to emerge that many of the actions of p53, including apoptosis and senescence, are mediated by miRNAs [79].

Aberrant epidermal growth factor receptor (EGFR) expression is known to lead to abnormalities in the normal cell growth process and initiate cancer. Increased EGFR expression is noted in several malignancies and it has become the focus of targeted therapies [80]. It is downregulated by miR-128, a miRNA that is frequently deleted in lung cancer. WeIss et al. [81] have shown that miR-128b loss of heterozygosity was frequent in tumour samples from 58 patients with NSCLC and correlated significantly with clinical response and survival following treatment with an EGFR-tyrosine kinase inhibitor.

\section{miRNAS AND THE IMMUNE RESPONSE}

The respiratory epithelium is constantly exposed to numerous foreign particles, including toxins and potential pathogens. The human immune system has developed into a highly complex and adaptable defence mechanism that protects us from these foreign and harmful substances. miRNAs have been shown to be pivotal in both the adaptive and innate immunity, including controlling the differentiation of various immune cell subsets as well as their immunological functions [16, 57, 82-91].

\section{miRNAs and the innate immune response}

The innate immune response provides the initial defence against infection by external pathogens and is predominantly mediated via myeloid cells such as macrophages, dendritic cells, monocytes and neutrophils. Several miRNAs, including miR155, miR-146 and miR-223, regulate the acute inflammatory response after recognition of pathogens by TLRs [92]. For example, miR-132, miR-146 and miR-155 are upregulated in macrophage cell lines in response to endotoxin [93]. miR-146 induction is triggered by TLRs that recognise bacterial constituents and by pro-inflammatory cytokines such as IL-1 $\beta$. It appears to provide an important feedback mechanism during severe inflammation by facilitating the resolution of inflammation [94]. This may involve miR-146-induced downregulation of key components relevant to the activation of the pro-inflammatory transcription factor nuclear factor (NF)- $\mathrm{BB}$. miR-155 is understood to be upregulated in macrophages as a consequence of exposure to a broad range of inflammatory mediators and viral and bacterial products $[44,95]$. Once induced, it regulates the release of inflammatory mediators and enhances the production of tumour necrosis factor (TNF)- $\alpha$ [95]. miR-155 also plays a vital role in host defence against infectious diseases, as it has been shown to modulate the maturation of dendritic cells (DC) and influences the ability of these professional antigen-presenting cells to bind pathogens by downregulating the expression of DC-specific intercellular adhesion molecule-3 grabbing non-integrin [96]. miR-223 has been found to play a crucial role in regulating granulocyte proliferation and activation [91, 97-99], although conflicting results exist as to its impact. Separate studies have identified miR-223 to be both a positive and negative regulator of granulocyte differentiation $[98,99]$. Despite these paradoxical results, miR-223 is felt to play an indispensable role in regulating inflammatory responses and 
this is supported by functional studies. Mice exposed to aerosolised endotoxin demonstrate a rapid increase in miR223 expression in lung and airway epithelia [100], while miR-223 knock-out mice spontaneously develop lung pathology and exaggerated tissue destruction after endotoxin challenge [97]. These results suggest that miR-223 probably also acts as a negative modulator of inflammatory responses in the lung.

\section{miRNAs and the adaptive immune response}

The adaptive immune response involves the selective recognition and removal of invading pathogens through the activation and subsequent clonal expansion of antigen-specific lymphocytes.

\section{T-cells}

miRNA expression varies across T-cell developmental stages and miRNAs also modulate the differentiation status of antigen-stimulated T-cells [101, 102]. miR-181 fine-tunes T-cell sensitivity and positive selection and regulates the quantitative levels of $\mathrm{T}$-cell response to antigens by modulating the signalling strength of the T-cell receptor [101, 103]. MiR-155 is also involved in T-cell differentiation and activation. In miR155-null mice, CD4+ T-cells show intrinsic bias towards a Thelper cell (Th) type 2 phenotype, with enhanced levels of the Th2 cytokines IL-4 and IL-5 [90]. In addition, miR-155 plays an important role in regulatory CD4 T-cell (Treg) generation and function [16]. Treg cells are CD4+ T-cells in charge of suppressing potentially deleterious activities of Th cells and their functions include maintaining self-tolerance and suppression of respiratory diseases such as asthma and allergy.

\section{B-cells}

Several studies have demonstrated that B-cells require miR-155 for normal production of isotype-switched, high-affinity antibodies and for a memory response [90, 104-106]. miR-155 knock-out mice fail to develop an immune response to pathogens following immunisation, supporting its role in antigen-driven B-cell maturation [90, 104].

\section{MIRNA AND CIGARETTE SMOKE}

Approximately 1.3 billion people smoke cigarettes worldwide and smoking is a significant risk factor for respiratory diseases including lung cancer and chronic obstructive pulmonary disease (COPD). Tobacco smoke has been shown to affect bronchial epithelial miRNA expression, with 28 miRNAs reported to be differentially expressed in current smokers in comparison to lifelong nonsmokers [47]. The majority of these were downregulated in the tobacco smokers. As several of these miRNAs (miR-218, miR-15a, miR-199b and miR-125b) have also been shown to be downregulated in other cancers, these changes, by implication, have potential relevance to the development of tobacco-related malignancies [76, 107-109].

Animal-based studies have also linked cigarette smoke exposure, miRNA alterations and cancer development. Rats exposed to cigarette smoke develop extensive alterations in pulmonary miRNA expression, with most of the observed alterations involving downregulation [110]. The most remarkably downregulated miRNAs belonged to families that regulate stress response, apoptosis, proliferation and angiogenesis, all pivotal processes in carcinogenesis. For example, cigarette smoke has been shown to downregulate the let-7 family of miRNAs. As described previously, let-7 has been found to be poorly expressed in human lung tumours, its inhibition results in an increased division of lung cancer cells in vitro [111], and lung cancer patients with low let-7 expression survive for a shorter time than those with high expression [76]. Cigarette smoke exposure in rodents has also been shown to downregulate several miRNAs involved in silencing angiogenic activities, providing evidence that cigarette smoke stimulates vessel growth in lungs [110]. It is noteworthy that these changes were evident before cancer onset, arising as an early response to environmental carcinogen exposure. Finally, in rodent studies cigarette smoke downregulated several miRNAs involved in the

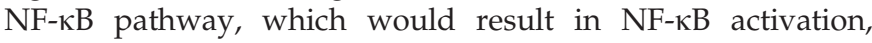
nuclear translocation and inflammation [110].

\section{miRNA and COPD}

COPD is a heterogeneous disease defined by airflow limitation that is not fully reversible and an abnormal persistent inflammatory response of the lung to noxious stimuli, particularly cigarette smoke [112]. The role of miRNAs in the altered immune responses and homeostatic mechanisms of COPD is beginning to emerge. 34 miRNAs were found to be differentially expressed in induced sputum from never-smokers compared with current smokers without COPD, while eight miRNAs were significantly reduced in current smokers with COPD compared to never-smokers (miR-34c, miR-218, miR-34b, let-7c, miR-3423p, miR-125a-5p, miR-30e-3p and miR-125b) [113]. Levels of let7c significantly inversely correlated with protein levels of TNF receptor type II (TNFR-II), which is implicated in the pathogenesis of COPD. In mouse models of COPD, knocking out TNFR-II protects against the development of cigarette-smoke induced inflammation and emphysema [114]. Intriguingly, compared to never-smokers, let-7c was only reduced in current smokers with COPD and not in ex-smokers with COPD or smokers without COPD, suggesting that let-7c is involved in the development of COPD due to smoking, but not in the persistence of airway inflammation following smoking cessation.

Expression profiling of lung tissue from subjects with COPD and smokers without COPD has revealed 70 miRNAs that are differentially expressed, including miR-223, miR-127a, miR424 and miR-15b [115]. The group concentrated on miR-15b and found that expression correlated with severity of COPD as assessed by spirometry [115]. Interestingly, miR-15b was able to regulate components of the transforming growth factor (TGF)- $\beta$ pathway, a key growth factor implicated in airway remodelling in COPD.

It is now accepted that abnormal repair responses are involved in the pathogenesis of COPD. Fibroblasts from patients with COPD have been shown to under-express miR-146a in response to inflammatory cytokines ex vivo, which leads to over-expression of cyclooxygenase- 2 and increased production of prostaglandin (PG) $E_{2}$ [116]. $\mathrm{PGE}_{2}$ is an inflammatory mediator that is known to be increased in the lungs of patients with COPD and is also a potent inhibitor of lung fibroblast repair functions [117, 118]. Cytokine-stimulated miR-146a expression also correlated with clinical severity of COPD as assessed by airflow limitation and diffusion capacity.

\section{ASTHMA}

Asthma is a common respiratory disease characterised by reversible airflow limitation, chronic airway inflammation and 
tissue remodelling. Both genetic factors and exposure to environmental triggers are involved in the pathogenesis of the disease, which is characterised by an abnormal Th2 response. Although studies are emerging that highlight the role of miRNAs in inflammatory processes, very few have specifically looked at their role in asthma pathogenesis. Work from our group has shown that miR-155 has a central role in the acquisition and maintenance of a Th2 phenotype by modulating the response of human macrophages to IL-13 [119]. IL-13 is a key Th2-derived effector cytokine considered to be relevant to asthma pathogenesis, and induces allergic airway inflammation. Inhibition of miR-155 was shown to lead to an increase in transcription factors involved in the generation of a Th2 microenvironment, implicating this miRNA in the pathogenesis of asthma. It has also been proposed that IL-13 may modulate the phenotype of bronchial smooth muscle cells by downregulating levels of miR-133a and resulting in increased expression of RhoA protein [120, 121]. RhoA is a key protein in smooth muscle contraction and its upregulation is associated with the augmented contraction of bronchial smooth muscle $[122,123]$.

One experimental study in a mouse model of asthma identified 21 miRNAs that were differentially expressed between mice with allergic airway inflammation and control mice [124]. In particular, over-expression of miR-21 and repression of miR-1 were evident in IL-13 transgenic mice - mice that overexpressed IL-13 in a lung-specific manner. miR-21 is thought to target IL-12, a macrophage-derived cytokine that is involved in adaptive immune responses involving Th1 cell polarisation. Increased miR-21 would lead to a decrease in IL-12 and may prime for Th2- and IL-13-associated responses. Downregulation of IL-12 has also been shown to affect Treg cell function, leading to a pro-inflammatory phenotype. miR-1 was identified as the most downregulated miRNA. It is considered to be a musclespecific miRNA and important for normal muscle physiology $[125,126]$. Downregulation of miR-1 has been associated with cardiac and skeletal muscle hypertrophy [127, 128], and it is interesting to speculate that it could also contribute to the smooth muscle hypertrophy and remodelling seen in asthma.

In a mouse model of house dust mite-induced allergic asthma, levels of miR-16, -21 and -126 have been reported to be significantly elevated in comparison to control animals. The relevance of miR-126 has also been suggested in a mouse model of acute allergic inflammation, in which the intranasal administration of antagomirs that inhibit miR-126 led to suppression of airway hyper-responsiveness to methacholine, attenuation of mucus hypersecretion and inhibition of eosinophil recruitment [129]. However, in the house dust mite sensitisation model, which is considered to reflect chronic asthma, the same treatment was only able to suppress eosinophil recruitment into the airways and had no effect on airway wall inflammation or remodelling [130]. This suggests and highlights that multiple miRNAs are likely to regulate chronic diseases such as asthma.

As mentioned previously, miR-146 is thought to play a key role in the negative feedback regulation of inflammation following activation by the innate immune system. Within the asthmatic airway miR-146 is thought to play a similar role. An increase in miR-146 levels has been reported to reduce the IL- $1 \beta$-induced
IL- 6 and IL- 8 release by both airway smooth muscle cells and alveolar epithelial cells [94, 131]. Importantly, this negative regulation was only observed at high IL-1 $\beta$ concentrations, suggesting that it might be an important feedback mechanism during severe inflammation.

Other groups have begun to look for differences in miRNA expression profiles between patients with asthma and normal controls. One such study investigated the expression of 227 miRNAs in bronchial biopsies of patients with mild asthma and non-asthmatic healthy controls and found that no miRNAs were differentially expressed [132]. Of interest, this study also demonstrated that corticosteroid treatment did not influence the expression of miRNAs despite improved lung function. It was noted that different cell types within the airways had different miRNA expression profiles, which raises the potential that differential miRNA expression may be occurring in the airways of asthmatics but within particular cell types.

\section{OTHER RESPIRATORY DISEASES}

Recently, miR-126 expression has been shown to be downregulated in cystic fibrosis $(\mathrm{CF})$ versus non-CF airway epithelial cells, which correlated with a reciprocal increase in the expression of its predicted protein target TOM1 (target of Myb1) [133]. The authors suggest that TOM1 may play an antiinflammatory role in CF lung and postulate that its increased expression may be an attempt to compensate for the high proinflammatory burden in this condition. TOM1 acts as a negative regulator of signalling pathways induced by IL-1 $\beta$ and TNF- $\alpha$ and over-expression of TOM1 inhibits NF- $\kappa \mathrm{B}$ activity [134].

Attention has focused on miR-21 with respect to pulmonary fibrosis. It has been found that miR-21 is upregulated in the lungs of mice with bleomycin-induced fibrosis and also in the lungs of patients with idiopathic pulmonary fibrosis [135]. Additionally, miR-21 has been shown to be upregulated by TGF- $\beta$, a key pathological mediator of fibrotic diseases, and functions in an amplifying circuit to enhance the pro-fibrogenic activity of TGF- $\beta 1$ and promote fibrotic lung diseases. MiR-21 inhibition significantly reduces collagen deposition in the lungs of bleomycin-treated mice, making it a potential target for developing novel therapeutics in treating fibrotic diseases. As might be anticipated, this is not the only abnormal regulatory miRNA reported in fibrotic lung disease, with another study identifying 18 miRNAs, including let-7d, that are significantly decreased in idiopathic pulmonary fibrosis [136]. TGF- $\beta$ can decrease the expression of let-7d, which results in epithelialmesenchymal transition in vitro and increased collagen deposition and alveolar septal thickening in mouse lungs in vivo. MiR155 is also intricately involved in epithelial-mesenchymal interactions in the lung and specifically attenuates inflammatory cytokine-mediated keratinocyte growth factor release by lung fibroblasts [137]. This supports a role for miR-155 in the pathogenesis of pulmonary fibrosis and suggests that altered miR-155 expression could also have important pathophysiological impacts during acute lung injury. Compared to agematched controls, miR-155 knock-out mice develop spontaneous remodelling of their airways, characterised by increased collagen deposition and smooth muscle mass [90]. Work from our laboratory has shown that over-expression of miR-155 attenuates TGF- $\beta$-induced expression of pro-fibrotic genes [138]. 


\section{CONCLUSION}

miRNAs have a fundamental role in gene regulation and have the capacity to modulate multiple gene pathways. They play crucial roles in a range of biological processes including embryogenesis, differentiation, proliferation, apoptosis, signal transduction and carcinogenesis. In recent years there has also been an explosion of reports on miRNA involvement in a range of human diseases, including diseases and malignancies of the lung. This has generated much interest in potentially utilising disease-specific miRNAs in therapeutics and as aids to diagnoses and prognoses; consequently it is crucial that appropriate and reliable research methods are used. We still only have a preliminary understanding of miRNAs and much is yet to be learned about the specific and intricate role of miRNAs in respiratory diseases. As a single miRNA could target several hundred genes, determining which miRNA-mRNA pairs to focus on for functional studies can be challenging and even once a molecular interaction is confirmed biological relevance must be demonstrated. This can be extremely arduous and timeconsuming because it is not the single miRNA-mRNA connection that needs to be studied but the effect of multiple miRNAs targeting a single gene of interest. Frequently in vitro systems are used to validate miRNA interactions and caution is required to circumvent erroneous interpretation of data. However, there is much interest in this exciting field and the successful translation of miRNA research is bound to deepen our understanding of the pathogenesis of diseases and influence the prevention, diagnosis, prognosis and therapy of respiratory diseases.

\section{STATEMENT OF INTEREST}

None declared.

\section{REFERENCES}

1 Claverie JM. Gene number. What if there are only 30,000 human genes? Science 2001; 291: 1255-1257.

2 Wright MW, Bruford EA. Naming "junk": human non-protein coding RNA (ncRNA) gene nomenclature. Hum Genomics 2011; 5: 90-98.

3 Lewis BP, Burge CB, Bartel DP. Conserved seed pairing, often flanked by adenosines, indicates that thousands of human genes are microRNA targets. Cell 2005; 120: 15-20.

4 Friedman RC, Farh KK, Burge CB, et al. Most mammalian mRNAs are conserved targets of microRNAs. Genome Res 2009; 19: 92-105.

5 Lee RC, Feinbaum RL, Ambros V. The C. elegans heterochronic gene lin-4 encodes small RNAs with antisense complementarity to lin-14. Cell 1993; 75: 843-854.

6 Lagos-Quintana M, Rauhut R, Lendeckel W, et al. Identification of novel genes coding for small expressed RNAs. Science 2001; 294: 853-858.

7 Williams AE. Functional aspects of animal microRNAs. Cell Mol Life Sci 2008; 65: 545-562.

8 Stefani G, Slack FJ. Small non-coding RNAs in animal development. Nat Rev Mol Cell Biol 2008; 9: 219-230.

9 Bartel DP. MicroRNAs: genomics, biogenesis, mechanism, and function. Cell 2004; 116: 281-297.

10 Mack GS. MicroRNA gets down to business. Nat Biotechnol 2007; 25: 631-638.

11 Care A, Catalucci D, Felicetti F, et al. MicroRNA-133 controls cardiac hypertrophy. Nat Med 2007; 13: 613-618.

12 Poy MN, Eliasson L, Krutzfeldt J, et al. A pancreatic islet-specific microRNA regulates insulin secretion. Nature 2004; 432: 226-230.
13 Fiore R, Siegel G, Schratt G. MicroRNA function in neuronal development, plasticity and disease. Biochim Biophys Acta 2008; 1779: 471-478.

14 Lu J, Getz G, Miska EA, et al. MicroRNA expression profiles classify human cancers. Nature 2005; 435: 834-838.

15 Krutzfeldt J, Stoffel M. MicroRNAs: a new class of regulatory genes affecting metabolism. Cell Metab 2006; 4: 9-12.

$16 \mathrm{Lu} \mathrm{LF}$, Liston A. MicroRNA in the immune system, microRNA as an immune system. Immunology 2009; 127: 291-298.

17 Denli AM, Tops BB, Plasterk RH, et al. Processing of primary microRNAs by the Microprocessor complex. Nature 2004; 432: 231-235.

18 Gregory RI, Yan KP, Amuthan G, et al. The Microprocessor complex mediates the genesis of microRNAs. Nature 2004; 432: 235-240.

19 Han J, Lee Y, Yeom KH, et al. The Drosha-DGCR8 complex in primary microRNA processing. Genes Dev 2004; 18: 3016-3027.

20 Engels BM, Hutvagner G. Principles and effects of microRNAmediated post-transcriptional gene regulation. Oncogene 2006; 25: 6163-6169.

21 Pillai RS, Bhattacharyya SN, Filipowicz W. Repression of protein synthesis by miRNAs: how many mechanisms? Trends Cell Biol 2007; 17: 118-126.

22 Guo H, Ingolia NT, Weissman JS, Bartel DP. Mammalian microRNAs predominantly act to decrease target mRNA levels. Nature 2010; 466: 835-840.

23 Schmittgen TD, Jiang J, Liu Q, et al. A high-throughput method to monitor the expression of microRNA precursors. Nucleic Acids Res 2004; 32: e43.

24 Peltier HJ, Latham GJ. Normalization of microRNA expression levels in quantitative RT-PCR assays: identification of suitable reference RNA targets in normal and cancerous human solid tissues. RNA 2008; 14: 844-852.

25 Deo A, Carlsson J, Lindlof A. How to choose a normalization strategy for miRNA quantitative real-time (qPCR) arrays. J Bioinform Comput Biol 2011; 9: 795-812.

26 Creighton CJ, Reid JG, Gunaratne PH. Expression profiling of microRNAs by deep sequencing. Brief Bioinform 2009; 10: 490-497.

27 Bentwich I, Avniel A, Karov Y, et al. Identification of hundreds of conserved and nonconserved human microRNAs. Nat Genet 2005; 37: 766-770.

28 Peter ME. Targeting of mRNAs by multiple miRNAs: the next step. Oncogene 2010; 29: 2161-2164.

29 Nana-Sinkam SP, Hunter MG, Nuovo GJ, et al. Integrating the MicroRNome into the study of lung disease. Am J Respir Crit Care Med 2009; 179: 4-10.

30 Kuhn DE, Martin MM, Feldman DS, et al. Experimental validation of miRNA targets. Methods 2008; 44: 47-54.

31 Schmittgen TD. Part B - microRNAs: microRNA methods. Methods 2008; 44: 1-2.

32 Breving K, Esquela-Kerscher A. The complexities of microRNA regulation: mirandering around the rules. Int J Biochem Cell Biol 2010; 42: 1316-1329.

33 Chang TC, Wentzel EA, Kent OA, et al. Transactivation of miR34 a by p53 broadly influences gene expression and promotes apoptosis. Mol Cell 2007; 26: 745-752.

34 He L, He X, Lim LP, et al. A microRNA component of the p53 tumour suppressor network. Nature 2007; 447: 1130-1134.

35 Raver-Shapira N, Marciano E, Meiri E, et al. Transcriptional activation of miR-34a contributes to p53-mediated apoptosis. Mol Cell 2007; 26: 731-743.

36 Brueckner B, Stresemann C, Kuner R, et al. The human let-7a-3 locus contains an epigenetically regulated microRNA gene with oncogenic function. Cancer Res 2007; 67: 1419-1423.

37 Thomson JM, Newman M, Parker JS, et al. Extensive posttranscriptional regulation of microRNAs and its implications for cancer. Genes Dev 2006; 20: 2202-2207. 
38 Wulczyn FG, Smirnova L, Rybak A, et al. Post-transcriptional regulation of the let-7 microRNA during neural cell specification. FASEB J 2007; 21: 415-426.

39 Lee EJ, Baek M, Gusev Y, et al. Systematic evaluation of microRNA processing patterns in tissues, cell lines, and tumors. RNA 2008; 14: 35-42.

40 Hutvagner G, McLachlan J, Pasquinelli AE, et al. A cellular function for the RNA-interference enzyme Dicer in the maturation of the let-7 small temporal RNA. Science 2001; 293: 834-838

41 Obernosterer $\mathrm{G}$, Leuschner PJ, Alenius $\mathrm{M}$, et al. Post-transcriptional regulation of microRNA expression. RNA 2006; 12: 1161-1167.

42 Tan Z, Randall G, Fan J, et al. Allele-specific targeting of microRNAs to HLA-G and risk of asthma. Am J Hum Genet 2007; 81: 829-834.

43 Nicolae D, Cox NJ, Lester LA, et al. Fine mapping and positional candidate studies identify HLA-G as an asthma susceptibility gene on chromosome 6p21. Am J Hum Genet 2005; 76: 349-357.

44 O'Connell RM, Taganov KD, Boldin MP, et al. MicroRNA-155 is induced during the macrophage inflammatory response. Proc Natl Acad Sci USA 2007; 104: 1604-1609.

45 Liu G, Friggeri A, Yang Y, et al. miR-147, a microRNA that is induced upon Toll-like receptor stimulation, regulates murine macrophage inflammatory responses. Proc Natl Acad Sci USA 2009; 106: 15819-15824.

46 Chiba $\mathrm{Y}$, Tanabe M, Goto K, et al. Down-regulation of miR-133a contributes to up-regulation of RhoA in bronchial smooth muscle cells. Am J Respir Crit Care Med 2009; 180: 713-719.

47 Schembri F, Sridhar S, Perdomo C, et al. MicroRNAs as modulators of smoking-induced gene expression changes in human airway epithelium. Proc Natl Acad Sci USA 2009; 106 2319-2324.

48 Kulshreshtha R, Ferracin M, Wojcik SE, et al. A microRNA signature of hypoxia. Mol Cell Biol 2007; 27: 1859-1867.

49 Zhang J, Zhang F, Didelot X, et al. Maternal high fat diet during pregnancy and lactation alters hepatic expression of insulin like growth factor-2 and key microRNAs in the adult offspring. BMC Genomics 2009; 10: 478.

50 Farh KK, Grimson A, Jan C, et al. The widespread impact of mammalian microRNAs on mRNA repression and evolution. Science 2005; 310: 1817-1821.

51 Stark A, Brennecke J, Bushati N, et al. Animal microRNAs confer robustness to gene expression and have a significant impact on 3’UTR evolution. Cell 2005; 123: 1133-1146.

52 Zeng Y. Principles of micro-RNA production and maturation. Oncogene 2006; 25: 6156-6162.

53 Williams AE, Moschos SA, Perry MM, et al. Maternally imprinted microRNAs are differentially expressed during mouse and human lung development. Dev Dyn 2007; 236: 572-580.

54 Williams AE, Perry MM, Moschos SA, et al. MicroRNA expression in the aging mouse lung. BMC Genomics 2007; 8: 172.

55 Harris KS, Zhang Z, McManus MT, et al. Dicer function is essential for lung epithelium morphogenesis. Proc Natl Acad Sci USA 2006; 103: 2208-2213.

$56 \mathrm{Lu} \mathrm{Y,} \mathrm{Thomson} \mathrm{JM,} \mathrm{Wong} \mathrm{HY,} \mathrm{et} \mathrm{al.} \mathrm{Transgenic} \mathrm{over-expression}$ of the microRNA miR-17-92 cluster promotes proliferation and inhibits differentiation of lung epithelial progenitor cells. Dev Biol 2007; 310: 442-453.

57 Ventura A, Young AG, Winslow MM, et al. Targeted deletion reveals essential and overlapping functions of the miR-17 through 92 family of miRNA clusters. Cell 2008; 132: 875-886.

58 Calin GA, Sevignani C, Dumitru CD, et al. Human microRNA genes are frequently located at fragile sites and genomic regions involved in cancers. Proc Natl Acad Sci USA 2004; 101: 2999-3004.

59 Wang QZ, Xu W, Habib N, et al. Potential uses of microRNA in lung cancer diagnosis, prognosis, and therapy. Curr Cancer Drug Targets 2009; 9: 572-594.
60 Johnson SM, Grosshans H, Shingara J, et al. RAS is regulated by the let-7 microRNA family. Cell 2005; 120: 635-647.

61 Esquela-Kerscher A, Trang P, Wiggins JF, et al. The let-7 microRNA reduces tumor growth in mouse models of lung cancer. Cell Cycle 2008; 7: 759-764.

62 Kumar MS, Erkeland SJ, Pester RE, et al. Suppression of nonsmall cell lung tumor development by the let-7 microRNA family. Proc Natl Acad Sci USA 2008; 105: 3903-3908.

63 Takamizawa J, Konishi H, Yanagisawa K, et al. Reduced expression of the let-7 microRNAs in human lung cancers in association with shortened postoperative survival. Cancer Res 2004; 64: 3753-3756

64 Weidhaas JB, Babar I, Nallur SM, et al. MicroRNAs as potential agents to alter resistance to cytotoxic anticancer therapy. Cancer Res 2007; 67: 11111-11116.

65 Hayashita Y, Osada H, Tatematsu Y, et al. A polycistronic microRNA cluster, miR-17-92, is overexpressed in human lung cancers and enhances cell proliferation. Cancer Res 2005; 65: 9628-9632.

66 Wang Y, Lee CG. MicroRNA and cancer - focus on apoptosis. J Cell Mol Med 2009; 13: 12-23.

67 Dews M, Homayouni A, Yu D, et al. Augmentation of tumor angiogenesis by a Myc-activated microRNA cluster. Nat Genet 2006; 38: 1060-1065.

68 Matsubara H, Takeuchi T, Nishikawa E, et al. Apoptosis induction by antisense oligonucleotides against miR-17-5p and $\mathrm{miR}-20 \mathrm{a}$ in lung cancers overexpressing miR-17-92. Oncogene 2007; 26: 6099-6105.

69 Mitchell PS, Parkin RK, Kroh EM, et al. Circulating microRNAs as stable blood-based markers for cancer detection. Proc Natl Acad Sci USA 2008; 105: 10513-10518.

70 Gilad S, Meiri E, Yogev Y, et al. Serum microRNAs are promising novel biomarkers. PLoS One 2008; 3: e3148.

71 Chen $\mathrm{X}, \mathrm{Ba} \mathrm{Y}, \mathrm{Ma} \mathrm{L}$, et al. Characterization of microRNAs in serum: a novel class of biomarkers for diagnosis of cancer and other diseases. Cell Res 2008; 18: 997-1006.

72 Hunter MP, Ismail N, Zhang X, et al. Detection of microRNA expression in human peripheral blood microvesicles. PLoS One 2008; 3: e3694.

73 Gallo A, Tandon M, Alevizos I, et al. The majority of microRNAs detectable in serum and saliva is concentrated in exosomes. PLoS One 2012; 7: e30679.

74 Lebanony D, Benjamin H, Gilad S, et al. Diagnostic assay based on hsa-miR-205 expression distinguishes squamous from nonsquamous non-small-cell lung carcinoma. J Clin Oncol 2009; 27: 2030-2037.

75 Yu SL, Chen HY, Chang GC, et al. MicroRNA signature predicts survival and relapse in lung cancer. Cancer Cell 2008; 13: 48-57.

76 Yanaihara N, Caplen N, Bowman E, et al. Unique microRNA molecular profiles in lung cancer diagnosis and prognosis. Cancer Cell 2006; 9: 189-198.

77 Markou A, Tsaroucha EG, Kaklamanis L, et al. Prognostic value of mature microRNA-21 and microRNA-205 overexpression in non-small cell lung cancer by quantitative real-time RT-PCR. Clin Chem 2008; 54: 1696-1704.

$78 \mathrm{Hu}$ Z, Chen J, Tian T, et al. Genetic variants of miRNA sequences and non-small cell lung cancer survival. J Clin Invest 2008; 118: 2600-2608.

79 Toledo F, Bardot B. Cancer: three birds with one stone. Nature 2009; 460: 466-467.

80 Pao W, Miller VA. Epidermal growth factor receptor mutations, small-molecule kinase inhibitors, and non-small-cell lung cancer: current knowledge and future directions. J Clin Oncol 2005; 23 2556-2568.

81 Weiss GJ, Bemis LT, Nakajima E, et al. EGFR regulation by microRNA in lung cancer: correlation with clinical response and 
survival to gefitinib and EGFR expression in cell lines. Ann Oncol 2008; 19: 1053-1059.

82 Vasilatou D, Papageorgiou S, Pappa V, et al. The role of microRNAs in normal and malignant hematopoiesis. Eur $J$ Haematol 2010; 84: 1-16.

83 Liang TJ, Qin CY. The emerging role of microRNAs in immune cell development and differentiation. APMIS 2009; 117: 635-643.

84 Navarro F, Lieberman J. Small RNAs guide hematopoietic cell differentiation and function. J Immunol 2010; 184: 5939-5947.

85 Kong KY, Owens KS, Rogers JH, et al. MIR-23A microRNA cluster inhibits B-cell development. Exp Hematol 2010; 38: 629-640.

$86 \mathrm{Lu} \mathrm{J}$, Guo S, Ebert BL, et al. MicroRNA-mediated control of cell fate in megakaryocyte-erythrocyte progenitors. Dev Cell 2008; 14: 843-853.

87 Ben-Ami O, Pencovich N, Lotem J, et al. A regulatory interplay between miR-27a and Runx1 during megakaryopoiesis. Proc Natl Acad Sci USA 2009; 106: 238-243.

88 Romania P, Lulli V, Pelosi E, et al. MicroRNA 155 modulates megakaryopoiesis at progenitor and precursor level by targeting Ets-1 and Meis1 transcription factors. Br J Haematol 2008; 143: 570-580.

89 Fontana L, Pelosi E, Greco P, et al. MicroRNAs 17-5p-20a-106a control monocytopoiesis through AML1 targeting and M-CSF receptor upregulation. Nat Cell Biol 2007; 9: 775-787.

90 Rodriguez A, Vigorito E, Clare S, et al. Requirement of bic/ microRNA-155 for normal immune function. Science 2007; 316: 608-611.

91 Chen CZ, Li L, Lodish HF, et al. MicroRNAs modulate hematopoietic lineage differentiation. Science 2004; 303: 83-86.

92 Bi Y, Liu G, Yang R. MicroRNAs: novel regulators during the immune response. J Cell Physiol 2009; 218: 467-472.

93 Taganov KD, Boldin MP, Chang KJ, et al. NF-אB-dependent induction of microRNA miR-146, an inhibitor targeted to signaling proteins of innate immune responses. Proc Natl Acad Sci USA 2006; 103: 12481-12486.

94 Perry MM, Moschos SA, Williams AE, et al. Rapid changes in microRNA-146a expression negatively regulate the IL-1 $\beta$ induced inflammatory response in human lung alveolar epithelial cells. J Immunol 2008; 180: 5689-5698.

95 Tili E, Michaille JJ, Cimino A, et al. Modulation of miR-155 and miR-125b levels following lipopolysaccharide/TNF- $\alpha$ stimulation and their possible roles in regulating the response to endotoxin shock. J Immunol 2007; 179: 5082-5089.

96 Martinez-Nunez RT, Louafi F, Friedmann PS, et al. MicroRNA155 modulates the pathogen binding ability of dendritic cells (DCs) by down-regulation of DC-specific intercellular adhesion molecule-3 grabbing non-integrin (DC-SIGN). J Biol Chem 2009; 284: 16334-16342.

97 Lindsay MA. MicroRNAs and the immune response. Trends Immunol 2008; 29: 343-351.

98 Fazi F, Rosa A, Fatica A, et al. A minicircuitry comprised of microRNA-223 and transcription factors NFI-A and C/EBP $\alpha$ regulates human granulopoiesis. Cell 2005; 123: 819-831.

99 Johnnidis JB, Harris MH, Wheeler RT, et al. Regulation of progenitor cell proliferation and granulocyte function by microRNA-223. Nature 2008; 451: 1125-1129.

100 Moschos SA, Williams AE, Perry MM, et al. Expression profiling in vivo demonstrates rapid changes in lung microRNA levels following lipopolysaccharide-induced inflammation but not in the anti-inflammatory action of glucocorticoids. BMC Genomics 2007; 8: 240.

101 Neilson JR, Zheng GX, Burge CB, et al. Dynamic regulation of miRNA expression in ordered stages of cellular development. Genes Dev 2007; 21: 578-589.

$102 \mathrm{Wu} \mathrm{H}$, Neilson JR, Kumar P, et al. miRNA profiling of naive, effector and memory CD8 T cells. PLoS One 2007; 2: e1020.
103 Li QJ, Chau J, Ebert PJ, et al. miR-181a is an intrinsic modulator of T cell sensitivity and selection. Cell 2007; 129: 147-161.

104 Thai TH, Calado DP, Casola S, et al. Regulation of the germinal center response by microRNA-155. Science 2007; 316: 604-608.

105 Teng G, Hakimpour P, Landgraf P, et al. MicroRNA-155 is a negative regulator of activation-induced cytidine deaminase. Immunity 2008; 28: 621-629.

106 Vigorito E, Perks KL, Abreu-Goodger C, et al. MicroRNA-155 regulates the generation of immunoglobulin class-switched plasma cells. Immunity 2007; 27: 847-859.

107 Calin GA, Liu CG, Sevignani C, et al. MicroRNA profiling reveals distinct signatures in B cell chronic lymphocytic leukemias. Proc Natl Acad Sci USA 2004; 101: 11755-11760.

108 Scott GK, Goga A, Bhaumik D, et al. Coordinate suppression of ERBB2 and ERBB3 by enforced expression of micro-RNA miR125a or miR-125b. J Biol Chem 2007; 282: 1479-1486.

109 Martinez I, Gardiner AS, Board KF, et al. Human papillomavirus type 16 reduces the expression of microRNA-218 in cervical carcinoma cells. Oncogene 2008; 27: 2575-2582.

110 Izzotti A, Calin GA, Arrigo P, et al. Downregulation of microRNA expression in the lungs of rats exposed to cigarette smoke. FASEB J 2009; 23: 806-812.

111 Johnson CD, Esquela-Kerscher A, Stefani G, et al. The let-7 microRNA represses cell proliferation pathways in human cells. Cancer Res 2007; 67: 7713-7722.

112 Rabe KF, Hurd S, Anzueto A, et al. Global strategy for the diagnosis, management, and prevention of chronic obstructive pulmonary disease: GOLD executive summary. Am J Respir Crit Care Med 2007; 176: 532-555.

113 Pottelberge GR, Mestdagh P, Bracke KR, et al. MicroRNA expression in induced sputum of smokers and patients with chronic obstructive pulmonary disease. Am J Respir Crit Care Med 2011; 183: 898-906.

114 D'hulst AI, Bracke KR, Maes T, et al. Role of tumour necrosis factor- $\alpha$ receptor p75 in cigarette smoke-induced pulmonary inflammation and emphysema. Eur Respir J 2006; 28: 102-112.

115 Ezzie ME, Crawford M, Cho JH, et al. Gene expression networks in COPD: microRNA and mRNA regulation. Thorax 2012; 67: 122-131.

116 Sato T, Liu X, Nelson A, et al. Reduced miR-146a increases prostaglandin $\mathrm{E}$ in chronic obstructive pulmonary disease fibroblasts. Am J Respir Crit Care Med 2010; 182: 1020-1029.

117 Montuschi P, Kharitonov SA, Ciabattoni G, et al. Exhaled leukotrienes and prostaglandins in COPD. Thorax 2003; 58: 585-588.

118 Togo S, Holz O, Liu X, et al. Lung fibroblast repair functions in patients with chronic obstructive pulmonary disease are altered by multiple mechanisms. Am J Respir Crit Care Med 2008; 178: 248-260.

119 Martinez-Nunez RT, Louafi F, Sanchez-Elsner T. The interleukin 13 (IL-13) pathway in human macrophages is modulated by microRNA-155 via direct targeting of interleukin 13 receptor $\alpha 1$ (IL13R $\alpha 1)$. J Biol Chem 2011; 286: 1786-1794.

120 Chiba Y, Nakazawa S, Todoroki M, et al. Interleukin-13 augments bronchial smooth muscle contractility with an upregulation of RhoA protein. Am J Respir Cell Mol Biol 2009; 40: 159-167.

121 Chiba $Y$, Todoroki M, Nishida $Y$, et al. A novel STAT6 inhibitor AS1517499 ameliorates antigen-induced bronchial hypercontractility in mice. Am J Respir Cell Mol Biol 2009; 41: 516-524.

122 Chiba Y, Sakai H, Misawa M. Augmented acetylcholine-induced translocation of RhoA in bronchial smooth muscle from antigeninduced airway hyperresponsive rats. Br J Pharmacol 2001; 133: 886-890.

123 Chiba Y, Ueno A, Shinozaki K, et al. Involvement of RhoAmediated $\mathrm{Ca}^{2+}$ sensitization in antigen-induced bronchial smooth muscle hyperresponsiveness in mice. Respir Res 2005; 6: 4. 
124 Lu TX, Munitz A, Rothenberg ME. MicroRNA-21 is up-regulated in allergic airway inflammation and regulates IL-12p35 expression. J Immunol 2009; 182: 4994-5002.

125 Rao PK, Kumar RM, Farkhondeh M, et al. Myogenic factors that regulate expression of muscle-specific microRNAs. Proc Natl Acad Sci USA 2006; 103: 8721-8726.

126 Chen JF, Mandel EM, Thomson JM, et al. The role of microRNA-1 and microRNA-133 in skeletal muscle proliferation and differentiation. Nat Genet 2006; 38: 228-233.

127 McCarthy JJ, Esser KA. MicroRNA-1 and microRNA-133a expression are decreased during skeletal muscle hypertrophy. J Appl Physiol 2007; 102: 306-313.

128 Luo X, Lin H, Pan Z, et al. Down-regulation of miR-1/miR-133 contributes to re-expression of pacemaker channel genes HCN2 and HCN4 in hypertrophic heart. J Biol Chem 2008; 283: 20045-20052.

129 Mattes J, Collison A, Plank M, et al. Antagonism of microRNA126 suppresses the effector function of $\mathrm{TH} 2$ cells and the development of allergic airways disease. Proc Natl Acad Sci USA 2009; 106: 18704-18709.

130 Collison A, Herbert C, Siegle JS, et al. Altered expression of microRNA in the airway wall in chronic asthma: miR-126 as a potential therapeutic target. BMC Pulm Med 2011; 11: 29.

131 Larner-Svensson $\mathrm{HM}$, Williams $\mathrm{AE}$, Tsitsiou $\mathrm{E}$, et al. Pharmacological studies of the mechanism and function of interleukin-1 $\beta$-induced miRNA-146a expression in primary human airway smooth muscle. Respir Res 2010; 11: 68.

132 Williams AE, Larner-Svensson H, Perry MM, et al. MicroRNA expression profiling in mild asthmatic human airways and effect of corticosteroid therapy. PLoS One 2009; 4: e5889.

133 Oglesby IK, Bray IM, Chotirmall SH, et al. miR-126 is downregulated in cystic fibrosis airway epithelial cells and regulates TOM1 expression. J Immunol 2010; 184: 1702-1709.

134 Yamakami M, Yokosawa H. TOM1 (target of Myb 1) is a novel negative regulator of interleukin-1- and tumor necrosis factorinduced signaling pathways. Biol Pharm Bull 2004; 27: 564-566.

135 Liu G, Friggeri A, Yang Y, et al. MiR-21 mediates fibrogenic activation of pulmonary fibroblasts and lung fibrosis. J Exp Med 2010; 207: 1589-1597.

136 Pandit KV, Corcoran D, Yousef $\mathrm{H}$, et al. Inhibition and role of let$7 \mathrm{~d}$ in idiopathic pulmonary fibrosis. Am J Respir Crit Care Med 2010; 182: 220-229.

137 Pottier N, Maurin $\mathrm{T}$, Chevalier B, et al. Identification of keratinocyte growth factor as a target of microRNA-155 in lung fibroblasts: implication in epithelial-mesenchymal interactions. PLoS One 2009; 4: e6718.

138 Louafi F, Martinez-Nunez RT, Sanchez-Elsner T. MicroRNA-155 targets SMAD2 and modulates the response of macrophages to transforming growth factor- $\beta$. J Biol Chem 2010; 285: 41328-41336. 